\title{
Integrating Virtual Reality with activity management for the treatment of
} fibromyalgia: Acceptability and preliminary efficacy

Azucena Garcia-Palacios ${ }^{1,2}$, Ph.D., Rocio Herrero ${ }^{1}$, Ph.D., Yolanda Vizcaíno ${ }^{1}$, B.A., Miguel Angel Belmonte ${ }^{3}$, M.D., Diana Castilla ${ }^{1}$, Ph.D., Guadalupe Molinari ${ }^{1}$, B.A., Rosa Maria Baños ${ }^{4,2}$, Ph.D., \& Cristina Botella ${ }^{1,2}$, Ph.D.

${ }^{1}$ Universitat Jaume I, Dpt. Psicología Básica, Clínica y Psicobiología. Spain.

${ }^{2}$ Ciber Fisiopatología Obesidad y Nutrición (CB06/03 Instituto Salud Carlos III, Spain).

${ }^{3}$ Hospital General de Castellón. Servicio de Reumatología, Spain

${ }^{4}$ Universidad de Valencia, Dept. Personalidad, Evaluación y Tratamientos Psicológicos, Spain.

Corresponding author:

Azucena Garcia-Palacios, PhD

Universitat Jaume I. Dpt. Psicologia Basica, Clinica y Psicobiologia

Avda Vicent Sos Baynat s/n 12071 Castellon. Spain

Phone: +34964387640

Fax: +34964729267

Email: azucena@uji.es

Copyright (C) 2014 Wolters Kluwer Health, Inc. Unauthorized reproduction of the article is prohibited. 


\section{Abstract}

Objectives: Cognitive-behavioral therapies (CBT) for fibromyalgia (FMS) are important interventions in the management of this condition. Empirical evidence reports that although the results are promising, further research is needed to respond more appropriately to these patients. This study focuses on exploring the use of Virtual Reality (VR) as an adjunct to the activity management component. The aim of this study is to present the results of a small-sized randomized controlled trial to test the preliminary efficacy and acceptability of this component.

Methods: The final sample was composed of 61 women diagnosed with FMS according to the American College of Rheumatology. The sample was randomly allocated to two conditions: VR treatment and treatment as usual.

Results: Participants in the VR condition achieved significant improvements in the primary outcome: disability measured with the FIQ. The improvement was also significant in secondary outcomes, such as perceived quality of life and some of the coping strategies included in the CPCI: Task persistence, and Exercise. There were no differences in other secondary outcome measures like pain intensity and interference and depression. Participants reported high satisfaction with the VR component.

Discussion: The effects were related to the psychological aspects targeted in the treatment. The component was well-accepted by FMS patients referred from a public hospital. These findings show that the VR component could be useful in the CBT treatment of FMS and encourage us to continue exploring the use of integrating VR with CBT interventions for the treatment of FMS. 
Key words: chronic pain, fibromyalgia, cognitive-behavioral therapy, activity management, information and communication technology, virtual reality.

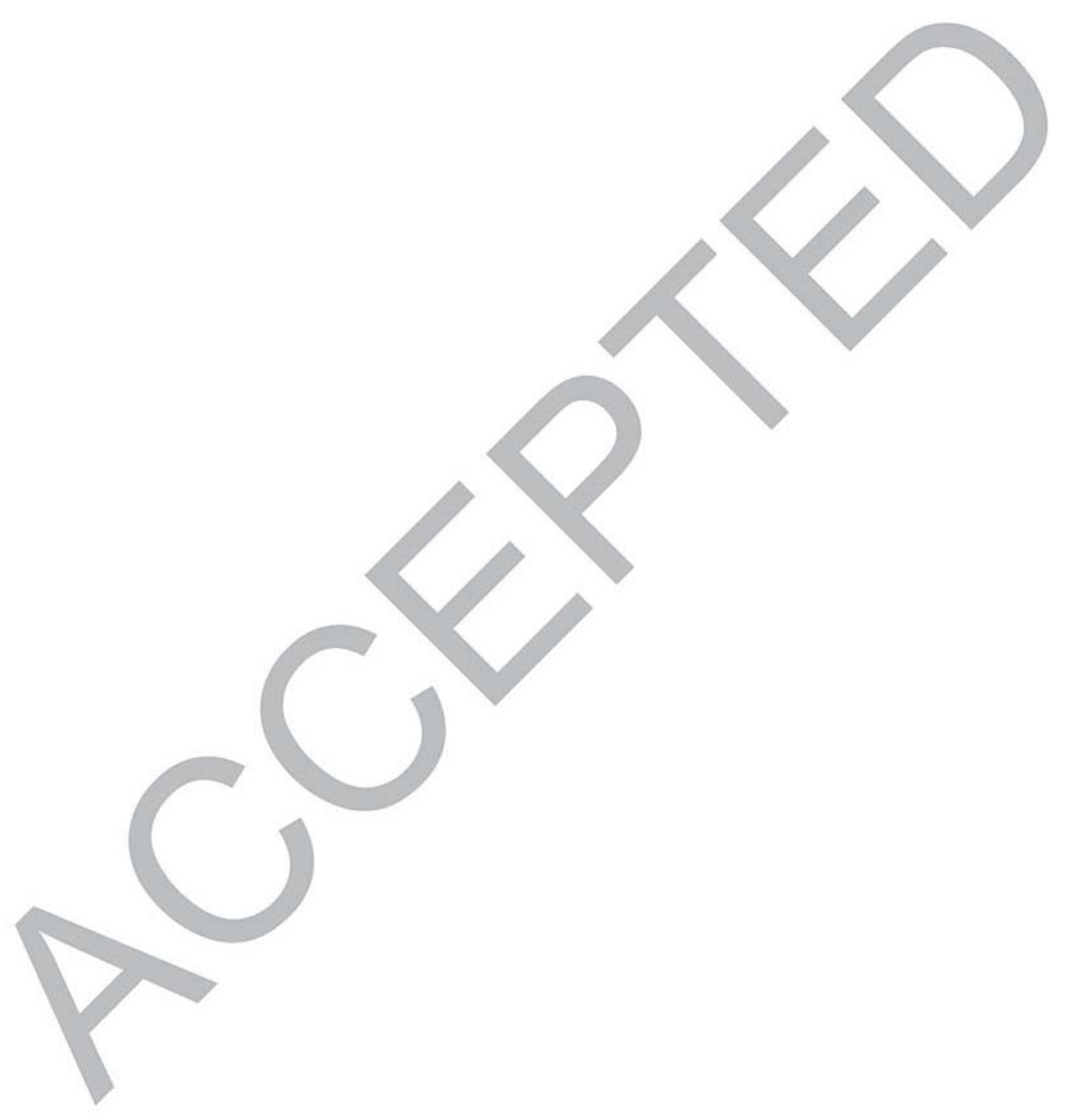

Copyright (C) 2014 Wolters Kluwer Health, Inc. Unauthorized reproduction of the article is prohibited. 


\section{Introduction}

Fibromyalgia syndrome (FMS) is a chronic musculoskeletal pain condition [1], characterized by widespread pain accompanied by fatigue and disturbed sleep and mood [2] affecting around 2 to $4 \%$ of the general population. [3] Comorbidities with affective and anxiety disorders are common [4] and it is associated with frequent medical consultation and work disability, resulting in high economic and social burden. [5]

The scientific literature supports the conceptualization of this condition in an integrative biopsychosocial model that includes physiological, psychological, and social aspects. [6] The treatment of FMS has a poor prognosis for recovery [7] and it is considered a challenge for health professionals. Addressing FMS from a multidimensional perspective seems to be more effective than from single approaches. [8]

The multidimensional perspective includes psychological programs as a promising treatment for FMS. Psychological aspects such as self-efficacy, attention, appraisal of pain, or avoidance are among the best predictors of disability caused by chronic pain. [9] There are several studies testing the efficacy of psychological programs for FMS. In an excellent metaanalysis, [10] 23 studies involving 1.396 patients were reviewed. The results revealed that the effect sizes of short- and long-term efficacy for psychological treatments were small yet robust. Cognitive Behavioral Therapy (CBT) was associated with the greatest effect sizes. The authors concluded that psychological programs are promising interventions for FMS. However, they noted that there are still relatively few studies on psychological treatments for FMS, and they recommended carrying out more efficacy studies. Another finding is that although the results are promising, there is still room for improvement given that effect sizes are only small or moderate. 
In summary, efficacy is still limited and further research is needed to respond more appropriately to patients with FMS.

Among the possible ways of improving psychological treatments in this field, an attractive line of research is the use of technology to support the interventions. One of the most promising technologies is Virtual Reality (VR). Its use in the field of psychology has increased in recent years. VR has been shown to be a useful tool in the treatment of several psychological disorders. [11;12] In the field of pain, VR has been used mainly as a distraction strategy for treating acute pain associated with medical procedures (e.g. wound care, physical therapy).

Pioneer work in this field was conducted by Dr. Hoffman's team at the University of Washington in studies that demonstrated the efficacy of VR for burn patients. [13] A systematic review about the use of VR distraction for pain reduction found promising results for the efficacy of this procedure. [14] Another use of VR in the treatment of acute pain is in combination with hypnosis. Patterson and his team $[15 ; 16]$ were the pioneers in this approach, finding important reduction in pain in burn patients.

When we look at the literature on the applications of VR in the field of chronic pain we see it is very scarce. Keefe et al. [17] have reviewed this issue and have found some preliminary studies focusing on VR for pain-related movement patterns and in combination with other psychological strategies. The idea is that VR could be a good tool to expose patients to movements that they may fear or avoid due to pain. It has been suggested that mirrors and VR could be potential new treatments for this condition. [18] A similar VR application has also been used to reduce phantom limb pain in amputees $[19 ; 20 ; 21]$ and in complex regional pain syndrome (CRPS). [22; 23] Gromala et al. [24] have developed a virtual walk that includes audio and visual feedback to induce relaxation while walking. There are some preliminary 
studies exploring the combination of VR with other interventions for persistent pain, such as a case study using VR hypnosis for neuropathic pain [25], a study protocol for the use of VR in combination with exposure to the treatment of pain catastrophizing in FMS [26] and VR as a distraction technique in chronic pain patients [27]. Our research group has explored the use of VR in combination with mindfulness and relaxation in a sample of six FMS patients. [28]

Keefe et al. [17] indicated some advantages of integrating VR with behavioral interventions for pain, like the potential of VR for standardizing the presentation of therapeutic instructions or the use of VR for patients who fail to respond to conventional interventions. They also mentioned the idea that once the patients have gained pain control via an integrated VR/behavioral intervention, they may be more motivated with practicing the strategies in their natural environment. We believe this is one of the best features of VR in the field of chronic pain. Keefe et al. finished their review encouraging researchers to be active in conducting studies exploring the possibilities of VR in chronic pain. In the present study, we explore the combination of VR with activity management.

Activity management is one of the most used treatment components in psychological programs for chronic pain. [29] An important goal for chronic pain patients is to balance rest and activity. However, this is a big challenge. First, patients have problems with pacing activity. After periods of restricted activity to diminish pain, patients may feel better, and then increase activity. This higher activity increases pain, and causes another period of inactivity, and this continues in a cyclical way. Unfortunately, what chronic pain patients learn from this pattern is to avoid activity. Second, patients with chronic pain attempt to protect and not use the parts of their body that hurt, which leads to an additional deactivation and muscle weakness. To address these problems the challenge is to identify the activities that the patient avoids, and to increase 
contact with valued activities. This can be achieved with activity management. This component consists of setting goals related to activities that are meaningful for the patient and progressively perform those activities balancing the periods of rest and activity. Feelings like low mood and fear, and the symptoms of the condition - pain, and more importantly, fatigue - get in the way of the willingness to perform the activities, causing low motivation and a poor sense of selfefficacy. An important goal is to promote motivation. The promotion of positive emotions can help to increase motivation towards activity. There is a line of research studying positive affect and their influences on cognitive processes, well-being and health, $[30,31]$ which offers evidence supporting the idea that positive affect promote flexible and creative thinking and play a main role in building psychological strength, and intellectual and social resources. [32] The promotion of positive emotions has several applications in the field of psychological treatments. For example, positive emotions have the ability to alter or reduce the effects of negative emotions; therefore promotion of positive affect may help in the regulation of negative emotions. Also, if positive affect promote the increase of available resources, its promotion might help to maintain long-term gains and provide people with strength to become more resilient. Our research team is working in the study of the effect of positive affect in the treatment of mental and health problems and in the use of technology to promote well-being (positive technology).

The VR component developed is aimed to make it easier for the patients to start and maintain an activity management program. Using a VR adaptive display we developed contents to motivate FMS sufferers to get involved in the activity management component. The aim of this study is to show the results of a small-sized randomized trial testing the efficacy and 
acceptability of the combination of VR with activity management. To our knowledge, this is the first study to test the use of VR in the field of chronic pain in a controlled study.

\section{Materials and Methods}

\section{Design}

A single-blind randomized controlled trial with repeated measures (pretreatment and posttreatment) and two conditions: VR activity management (VRAM) and treatment as usual (TAU). Missing data due to drop out were addressed using the last observation carried forward (LOCF) method.

\section{Participants}

Participants were recruited from the Rheumatology Service of a public hospital (Hospital General de Castellon, Spain). Some inclusion criteria were established - participants were required to: 1) have been diagnosed with FMS by a rheumatologist and in accordance with the American College of Rheumatology criteria; [1] 2) be 18-70 years old; 3) not be suffering from severe mental disorders such as schizophrenia, bipolar disorder, mental retardation, substance abuse or dependence, nor a mental disorder in need of immediate treatment (i.e. severe major depressive disorder); 4) not be suffering from a physical disease that could interfere with receiving a psychological treatment; and 5) not be in the process of requesting or suing for disability.

This research belongs to a funded project approved by an ethical review board. 
Ninety-five participants were eligible, but 34 were not allocated to the conditions for several reasons (see flow diagram in Figure 1). The main reason for the nine dropouts before allocation was that the participants found that if they would be allocated to the VRAM condition, the treatment sessions involved too much time (twice a week) and they were not ready to commit to it. The final sample comprised 61 Caucasian women with a diagnosis of FMS. The mean age of the sample was 50.48 years old $(S D=9.78)$, ranging from 23 to 70 , and the mean duration of the FMS diagnosis was 9.32 years $(S D=8.15)$. All participants agreed to participate in the study and signed an informed consent form. One participant dropped out from the VRAM condition because she did not find the treatment helpful. One participant dropped out from the TAU condition. She did not attend a programmed visit with the rheumatologist and did not give any reason for not showing up. She did not contact the rheumatology service to reschedule another visit.

- Figure 1 to be inserted here-

Most participants were married (78.7\%), 9.8\% were single, 9.9 divorced and 1.6 widow. Regarding education, $21.3 \%$ had less than eight years of education, $36.1 \%$ had an elementary level of education (eight years), 31.2\% had completed high school, and $11.4 \%$ had a university degree. As for occupation, $49.2 \%$ were housewives, $47.5 \%$ were employed outside of the home, and $3.3 \%$ were unemployed. Although $90 \%$ of the sample had a history of mood or anxiety disorders, only one patient had previously received psychological treatment. At the time of entering the study the participants' mood or anxiety disorders were in remission (they did not meet DSM-IV-TR criteria for any mood or anxiety disorders) as established by a structured diagnostic interview (SCID-I). [34] Most patients (49.2\%) were taking stable doses of painkillers such as paracetamol or non-steroidal anti-inflammatory drugs (NSAIDs). Forty-one percent were 
receiving stable doses of pregabalin and $9.8 \%$ were not taking any medication. Patients in the VR treatment condition were told not to change the type of medication they were taking or to increase dosages while participating in the study unless instructed by a rheumatologist. None of them made medication alterations for the duration of the study.

\section{Primary outcome variable}

Disability

Fibromyalgia Impact Questionnaire: [35; 36]: This is the most used self-report in the field of FMS. It assesses physical functioning, work status, depression, anxiety, sleep, pain, stiffness, fatigue, morning tiredness and well-being in patients with FMS. The FIQ is composed of ten questions, with each question rated on a four-point Likert-type scale. This instrument has demonstrated good psychometric properties across cultures and languages, including Spanish.

\section{Secondary outcome variables}

Pain intensity and interference

The Brief Pain Inventory (BPI): [37; 38] This instrument assesses the severity of pain and the impact of pain on daily functions. The BPI has demonstrated both reliability and validity across cultures and languages, including Spanish, and has been widely used for clinical pain assessment. It contains 11 items: the first four items refer to pain intensity and the following seven items relate to pain interference with activities.

\section{Coping}

Chronic Pain Coping Inventory (CPCI): [39] This instrument identifies coping strategies that patients use to deal with chronic pain. It is a 64-item measure that was designed to assess 
coping subscales that fall into three categories: illness-focused coping (Guarding, Resting, and Asking for Assistance), wellness-focused coping (Relaxation, Task Persistence, Exercise/Stretch, and Coping Self-Statements), and neutral coping (Seeking Social Support subscale). Subscales demonstrate adequate to good internal consistency and validity.

\section{Mood}

Beck Depression Inventory II (BDI-II): [40; 41] This is one of the most used self-report instruments to assess depression. It presents good psychometric properties in the English and Spanish versions. This inventory includes 21 items evaluating cognitive, behavioral, affective and somatic symptoms of depression. A score from 0 to 13 indicates minimal depression, from 14 to 19 mild depression, from 20 to 28 moderate depression, and from 29 to 63 severe depression.

Perceived quality of life

The Quality of Life Index (QLI-Sp) [42] is a self-report questionnaire that measures perceived health-related quality of life that includes 10 items or domains: physical well-being, psychological/emotional well-being, self-care and independent functioning, occupational functioning, interpersonal functioning, social-emotional support, community and services support, personal fulfillment, spiritual fulfillment, and overall quality of life.

Acceptability and satisfaction

Given that VR is a new element in the treatment of chronic pain we were interested in having the participants' opinion about it in order to assess satisfaction and acceptability. We used a scale (satisfaction and acceptability scale), adapted from Borkovec and Nau, [43] to measure 
satisfaction with the treatment program and with the VR component only after completion of the treatment. Participants were asked several questions about their satisfaction and acceptability regarding the treatment program and the VR component (in the results section the specific questions are displayed). The participants rated the items on a 0 to 10 scale where 0 was "not at all" and 10 was "completely."

\section{Treatment}

The treatment was a group CBT program for FMS supported by VR as an adjunct to the activity pacing component. The treatment was designed according to the guidelines of existing CBT programs. [29] The aim of this study was to test the acceptability and preliminary efficacy of the activity management component supported by VR. Because of that we only included the activity management component as the key ingredient of the program, and we did not include other useful components like cognitive therapy or relaxation. The program included six two-hour group sessions delivered twice a week. The session format was as follows: 1) Overview of the last session and introduction of agenda; 2) Homework review; 3) Specific content of session; 4) Homework assignment.

The treatment program included the following components:

1) Education about chronic pain and FMS: Participants were given descriptions of the biopsychological model of chronic pain and FMS, the psychological, biological and social factors influencing the experience of pain and other symptoms, and the rationale of the CBT program for the treatment of FMS. This component was administered in the first session.

2) Activity management: Participants were provided with the rationale for activity pacing (balancing activity with periods of rest by means of meaningful programmed activities in order 
to achieve a healthy level of activity, promoting self-efficacy, and decreasing negative affect and avoidance of activities because of pain). This component was administered from session 2 to 6 and was supported by a VR environment that was displayed on a big screen. The aim of this component was to use multimedia elements to enhance motivation and positive affect related to the performing of meaningful activities (see more detailed description in the section describing the virtual environment).

3) Relapse prevention: Participants were offered an overview of the program to help solve their doubts and to answer their questions about the different strategies they had learned. They were also assisted in planning how to use the strategies in the future and were instructed on how to anticipate and confront high-risk situations. Relapse prevention was administered in the last hour of session 6 .

Participants in the TAU condition received the usual care in a rheumatology unit in a public setting in Spain that consists of follow-up sessions by a rheumatologist to review the medication treatment. All participants attended at least 1 session in the five weeks after the pretreatment assessment.

\section{Therapists}

Four therapists participated in the study. There were two therapists in each group. The role of the leading therapist was to apply the treatment. The role of the assistant therapist was to prepare all the materials needed for each session, run the VR equipment, and to support the therapist in applying the treatment. The leading therapists held a Ph.D. degree and had expertise in the delivery of CBT and VR therapy. The other two therapists acted as assistant therapists. They were PhD students and they had a master degree and training in CBT and VR therapy. 


\section{Virtual environment description}

The configuration used different devices: Two PC computers, a 3 x 4 m screen made of reflective material, two projectors (with a 1920x1080 pixel resolution, 2000 lumen power and 50000:1 contrast) and a 7.1 surround audio system. The PC1 has the graphical outputs from its graphic card connected to the projector, which are used to project the environment on a metacrilate screen. Specifically, the main characteristics of this PC1 are the following: a $2 \mathrm{Ghz}$ Intel Pentium 4 processor, a $256 \mathrm{MB}$ graphic card, 1 GB RAM, an Ethernet network card for connecting to the other PC. The operating system was Windows XP.

The PC2 is the host of the therapist's application and controls the features of the VR environment that is shown to the patient. The characteristics of this PC2 are the following: a 3 Ghz Intel Pentium 4 processor, a Nvidia GeForce 7800 graphic card, 2 GB RAM, and a TFT screen.

The characteristics of this VR environment configuration (using a large screen and not a headmounted display) allow VR sessions to be performed in groups. This is important in the case of FMS given that group therapy is recommended for chronic pain sufferers. [29] Participants were placed in front of the screen in groups of six. One of the therapists was in charge of operating the VR system during the VR session.

The VR environment was an adaptive display named EMMA, developed in the framework of an EU-funded project (IST-2001-39192-EMMA, Engaging Media for Mental Health Applications). EMMA is a flexible VR environment that includes five predefined scenarios. These scenarios are aimed at promoting emotions and motivation, depending on the goal of the therapy session. The five scenarios are: a desert, a beach, a forest, a snowy landscape and a meadow. It is possible to 
use different elements in these scenarios, such as changes in the weather or in the time (day and night), and to include sounds, images, and videos. This VR environment has demonstrated its capacity to induce several emotions [44], and it has been used for the treatment of stress-related disorders. [45; 46]. We conducted a study to test its capacity to test positive emotions in chronic pain patients. [47]

In this study the scenarios chosen were the beach and the meadow. In addition, specific music, sounds, narratives and images were selected to encourage the occurrence of positive emotions and motivation in order to enhance self-efficacy and behavior activation. In Figures 2 and 3 we display images of the setting and the VR environment.

The music was selected following two methods. On the one hand, melodies were chosen following two rules of music structure: tempo and mode. [48; 49] A fast tempo evokes energy and activation, and a major mode is related with positive mood. $[50 ; 51]$ On the other hand, resources from the International Affective Digitized Sounds (IADS) [52] were included that take into consideration the dimensions of emotional arousal and valence, $[53 ; 54]$ and melodies with positive valence and high arousal were selected. With regard to the images, they were selected from the International Affective Picture System (IAPS) [55; 56], also meeting the criteria of positive valence and high arousal. Other images from other sources were also included, and were selected according to three dimensions: color, brightness and saturation. Research indicates that positive emotions are evoked through bright and high-saturated color images. Also, there was a predominance of green and blue, colors that have been shown to induce positive emotions. [57] 
In addition to images and sounds, we included narratives especially designed to promote motivation in FMS sufferers. The narratives were designed by a team of therapists with expertise in cognitive-behavior therapy and in the treatment of chronic pain.

With all these elements we designed three VR sessions that lasted around 20 minutes each. The use of VR was embedded in the activity management component. The content of the first VR session (VR1) included the rationale of the activity management and instructions to enhance motivation to start to perform meaningful activities. The second VR session (VR2) dealt with acknowledging and overcoming the barriers that prevent the performance of activities.

Finally, the third VR session (VR3) included instructions to acknowledge personal strengths that could be used to motivate patients to get involved in meaningful activities. VR1 was used in session 2 of the program, VR2 was used in sessions 3 and 5, and VR3 was used in sessions 4 and 6.

- Figures 2 and 3 to be inserted here-

\section{Procedure}

The present study was conducted at Universitat Jaume I in Castellon, Spain. One of the rheumatologists from the larger public hospital in the area (Author M. A. B.) referred FMS patients in need of psychological treatment to the study. Participants visited the university clinical psychology center and were evaluated for the inclusion criteria in a screening session and a diagnostic session where they completed the SCID. Participants were given information about the study and were asked to sign an informed consent form if they were willing to participate. The participants filled out the pretreatment assessment protocol in one assessment session with the help of a clinician. Next, using a free software tool named Random Allocation Software 2.0 (http://random-allocation-software.software.informer.com/2.0), they were randomly allocated to 
one of the experimental conditions. Participants in the VR treatment condition received the treatment program over three weeks. Three weeks after the completion of the treatment they were assessed with the posttreatment protocol. Participants in the treatment as usual (TAU) condition received the usual care provided in the rheumatology unit which only included visits with the rheumatologist and did not involve any psychological intervention. They were assessed again after five weeks (posttreatment assessment) and they were then offered CBT treatment in our university clinic.

\section{Results}

\section{Pretreatment comparisons}

Chi-square tests showed no differences between the groups at pre-test in any of the demographic variables: education, marital status and occupation. A student t-test revealed no differences between the groups regarding age.

Student t-tests were conducted to explore possible differences between the experimental conditions at pretreatment. There were no statistically significant differences between the groups in any of the outcome variables: impact of fibromyalgia (FIQ), depression (BDI-II), pain intensity and interference (BPI), quality of life (QLI), and coping strategies measured by the CPCI.

\section{Pre-post comparisons}

We conducted $2 \times 2$ repeated measures ANOVAs (being time the with-in subject variable and condition the between subject variable) in order to explore the efficacy of the VR intervention. 
We calculated Cohen $\mathrm{d}$ for an estimation of the effect size. In Table 1 the mean, standard deviation and Cohen $\mathrm{d}$ are displayed for the pre-post comparisons.

- Table 1 to be inserted here-

Regarding the primary outcome measure, the impact of fibromyalgia measured by the FIQ, we found a time effect $F(1,59)=23.99, p<0.001$ and a time x group interaction $F(1,59)=11.37, p$ $<0.01$. Participants in the VRAM group reported a significant reduction in the FIQ score compared to participants in the TAU condition. The effect size of the pre-post comparison in the VRAM group was large.

Secondary outcome measures were pain intensity and interference, depression, and perceived quality of life.

With regard to pain intensity, no significant time, $F(1,59)=2.26, p=0.138$, or interaction effect $F(1,59)=0.18, p=0.675$ were found. The same results were found regarding pain interference - no time, $F(1,59)=0.95, p=0.334$, nor interaction effects, $F(1,59)=2.18 ; p=0.145$.

The ANOVA did reveal a significant time effect, $F(1,59)=4.72, p=0.034$, but not an interaction effect, $F(1,59)=1.64, p=0.205$, regarding depression.

With regard to perceived quality of life, there was a significant time effect $F(1,59)=27.03$; $p<$ 0.001 , and interaction effect, $F(1,59)=15.35, p<0.001$. In this case, the analyses revealed a higher increase in this measure in the VRAM group compared to the TAU group. The pre-post comparison in the VRAM condition obtained a large effect size.

We were also interested in exploring changes in coping strategies from pretreatment to posttreatment. 
First we explored the illness-focused coping, Guarding, Resting, and Asking for assistance. A significant time effect, $F(1,59)=15.79, p<0.001$, and a non significant interaction effect, $F(1$, $59)=2.53, p=0.117$, was found for Guarding. Participants in the VRAM condition reported a reduction in the use of this coping strategy with a moderate effect size. In the TAU condition there was also a reduction but with a small effect size. No time, $F(1,59)=0.071, p=0.791$, or interaction effect, $F(1,59)=3.46, p=0.068$ was found for Resting. Similar results were obtained for Asking for help, no time effect, $F(1,59)=1.260, p=0.266$, nor interaction effect, $F(1,59)=0.256, p=0.615$.

As for the wellness-focused coping, regarding Relaxation, there was a time effect, $F(1,59)=$ 4.585, $p<0.05$, but not an interaction effect, $F(1,59)=0.832, p=0.366$. With regard to Task persistence, there was a significant time effect, $F(1,59)=13.20, p<0.01$, and a significant interaction effect, $F(1,59)=11.20, p<0.01$, indicating a larger increase in the use of this strategy in the VRAM condition (with a large effect size) compared to the TAU condition. Similar results were obtained in Exercise, a time effect, $F(1,59)=6.05, p<0.02$, and an interaction effect, $F(1,59)=6.56, p<0.02$, indicating a larger increase in this strategy in the VRAM condition, with a moderate effect size. A significant time effect was found regarding Coping self-statements, $F(1,59)=8.19, \mathrm{p}<0.02$, but not an interaction effect, $F(1,59)=1.633$, $p=0.206$.

Finally, the neutral coping strategy, Seeking social support did not reveal any time, $F(1,59)=$ $0.731, p=0.396$, or interaction effect, $F(1,59)=3.836, p=0.055$.

\section{Satisfaction and acceptability}

Copyright (C) 2014 Wolters Kluwer Health, Inc. Unauthorized reproduction of the article is prohibited. 
In Table 2 the results from the Satisfaction and Acceptability scale are displayed. The mean ratings given by the participants were very high. They found the treatment logical and useful. Participants in the VRAM condition were highly satisfied with the treatment and they will definitely recommend the treatment to a friend. The participants did not find the treatment aversive or unpleasant. Finally, regarding the specific VR component, they found it highly useful and reported high satisfaction with it.

- Table 2 to be inserted here-

\section{Discussion}

This work reports the results from a small-sized randomized controlled trial evaluating the feasibility, acceptability, and preliminary efficacy of a CBT program for FMS, including an activity management component supported by VR. We compared a treatment as usual condition (TAU) with a treatment condition including VR as an adjunct to the activity management component (VRAM).

The findings suggest that the VR treatment had positive effects in reducing the impact of FMS on the life of the participants (measured by the FIQ). The VRAM group also reported a significant improvement in perceived quality of life. In these two variables the effect sizes (Cohen $d$ ) from pretreatment to posttreatment were large. No significant changes were noted regarding pain intensity and interference, and depression. Regarding this last variable it is important to notice that participants in our sample did not report high levels of depression at pretreatment (in fact, none of them met DSM-IV criteria for depressive disorders). This could have influenced the lack of significant changes from pretreatment to posttreatment. 
Significant changes in the use of several coping strategies were found mainly in the wellnessfocused strategies. There were not significant differences between conditions in Guarding, Resting and Asking for Assistance, although the most significant effects were found in Guarding (medium effect size in the comparison between pretreatment and posttreatment). This strategy refers to the extent to which an individual restricts movements as a way of coping with pain. Restriction of movements is not recommended in chronic pain management because it increases pain, stiffness and other symptoms. It seems that the treatment program, focusing on activity activation, helped the participants to understand this fact and to start reducing this unhealthy strategy. Important changes occurred regarding wellness-focused strategies (Task persistence and Exercise). In this case, significant increments with medium to large effect sizes in the use of these two coping strategies were found in the VRAM group. There were no significant differences between conditions in the other healthy and neutral strategies (Relaxation, Coping self-statements and Seeking social support). The treatment program was focused on activity management which involves behaviour activation, restoring discontinued activities, and balancing activity with periods of rest. This is achieved by means of meaningful programmed activities. The goal is to maintain a healthy level of activity, promoting self-efficacy, and decreasing negative affect and avoidance of activities because of pain. Our data support that the work done during the treatment in activity management promoted the increase of Exercise (physical activity) and Task persistence (extent to which an individual continues normal activity despite the pain). Our program did not include other components like cognitive therapy, relaxation or communication skills. This could explain the lack of more significant effect in Coping self-statements (use of adaptive cognitions to cope with pain), Relaxation, or Seeking 
social support (frequency in which an individual seeks out a significant other for support when in pain).

Our findings support the importance of the activity management component in the treatment of FMS. [29] The treatment program included this component as the main therapeutic ingredient but not other psychological components such as cognitive therapy. The reason for doing so was that we were interested in exploring the feasibility of integrating VR with this component and making a preliminary evaluation of its effect without the influence of other powerful elements like formal cognitive therapy.

Working in this component produced a reduction in disability and an increase in the perceived quality of life. Restoring and balancing activity is a challenge in chronic pain and FMS. It is difficult for FMS sufferers to set goals related to activities that are meaningful for them and to perform those activities in a progressive way. Low mood, fear, pain and fatigue get in the way of the willingness to perform the activities, causing low motivation and a poor sense of selfefficacy. Our goal was to develop a VR environment with contents aimed to promote motivation and positive affect in order to support the activity management component. This is in line with the ideas reported by Keefe et al. [17] about the advantages of integrating VR with behavioral interventions for pain, such as the fact that VR offers the possibility of standardizing the presentation of therapeutic instructions. In our case we developed three different VR sessions for FMS that were offered in the same way to all participants in the VRAM condition and that could be used in the future for other FMS patients. VR is also a useful means for the patient to practice in a protected environment in order to be more motivated with practicing the strategies in their natural environment. In our case, the idea was to promote motivation and self-efficacy in VR to make it more likely and easier for the patients to perform the programmed activities. 
Innovation in technology and treatment development is a challenge involving several issues. One of the most important in the field of VR therapeutic applications is the acceptability of the technology. We evaluated this issue by asking patients to rate some questions related to their satisfaction with the treatment program and with the VR environments. These data are very promising because, although our sample was referred from a public hospital and had a low expertise in the use of technology in general, and no experience with the use of VR, they reported being very satisfied with the VR intervention. This is in line with other domains where VR has been applied. [12] This technology is usually well-accepted by patients with psychological disorders. Our data suggest that VR could also be well accepted by chronic pain sufferers. These data support the feasibility of incorporating this technology to real clinical settings. Also, the hardware and software needed to apply the VRAM component is not costly and the logistics is not complicated (two PCs, a $3 \times 4 \mathrm{~m}$ screen, two projectors and a 7.1 surround audio system could be installed in a regular room for group therapy).

Although the results from this study are promising, it has several limitations. The sample size was small and this study needs to be performed with larger samples. Another limitation is that we did not compare the VRAM condition with an active treatment condition or an attentioncontrol condition. The reason for this is that this is a preliminary test. The scarce literature in the use of VR in FMS and also in chronic pain took us to a less strict design for this first small-sized controlled study, with the aim of exploring the feasibility, acceptability and preliminary efficacy of VR in this population. Also, we chose the more ecological setting, a rheumatology unit at a public hospital where FMS in Spain usually are referred and treated. The disadvantage is that it is complicated to conduct a clinical trial in that setting and because of that we included only two experimental conditions. FMS sufferers in a public setting in our country are usually middle-age 
women with a low level of education and low expertise with technology. In our case, 57.4\% of the sample had eight or less years of education, and only $11.4 \%$ had a university degree. We had a previous experience using VR with this population in a case series with six participants, [28] but we were concerned with the acceptability of the technology by this population. Our data support the fact that the VR environment was well accepted by this study's sample. The next step is to conduct a clinical trial comparing the efficacy of a traditional CBT program with a CBT program including the VRAM component.

One more limitation is that the treatment program did not include other powerful psychological components usually included in CBT programs for FMS and did not include follow-up assessments. The reason for not doing so was that our aim was to explore the preliminary effect of the integration of VR in an activity management component. If we had included other components like cognitive therapy it would be more difficult to attribute the effect to the activity management program. In the future, if the utility of this component is confirmed, we plan to include our VR + activity management component in a multicomponent CBT program and test its short and long-term efficacy.

In summary, to our knowledge, this is the first controlled study of a preliminary evaluation of the utility, efficacy and acceptability of VR in the field of FMS and chronic pain. The findings are promising and indicate the potential of this technology in the treatment of chronic pain.

\section{Acknowledgments}

The research presented in this paper was funded in part by Fundació La Marató de TV3 (Ajuts de la Marató de TV3 2006), Ministerio de Educación y Ciencia. Spain. PROYECTOS 
CONSOLIDER-C (SEJ2006-14301/PSIC), Fundació Caixa Castelló-Bancaixa (P11B2009-30), and by Generalitat Valenciana, Redes de Excelencia ISIC (ISIC/2012/012).

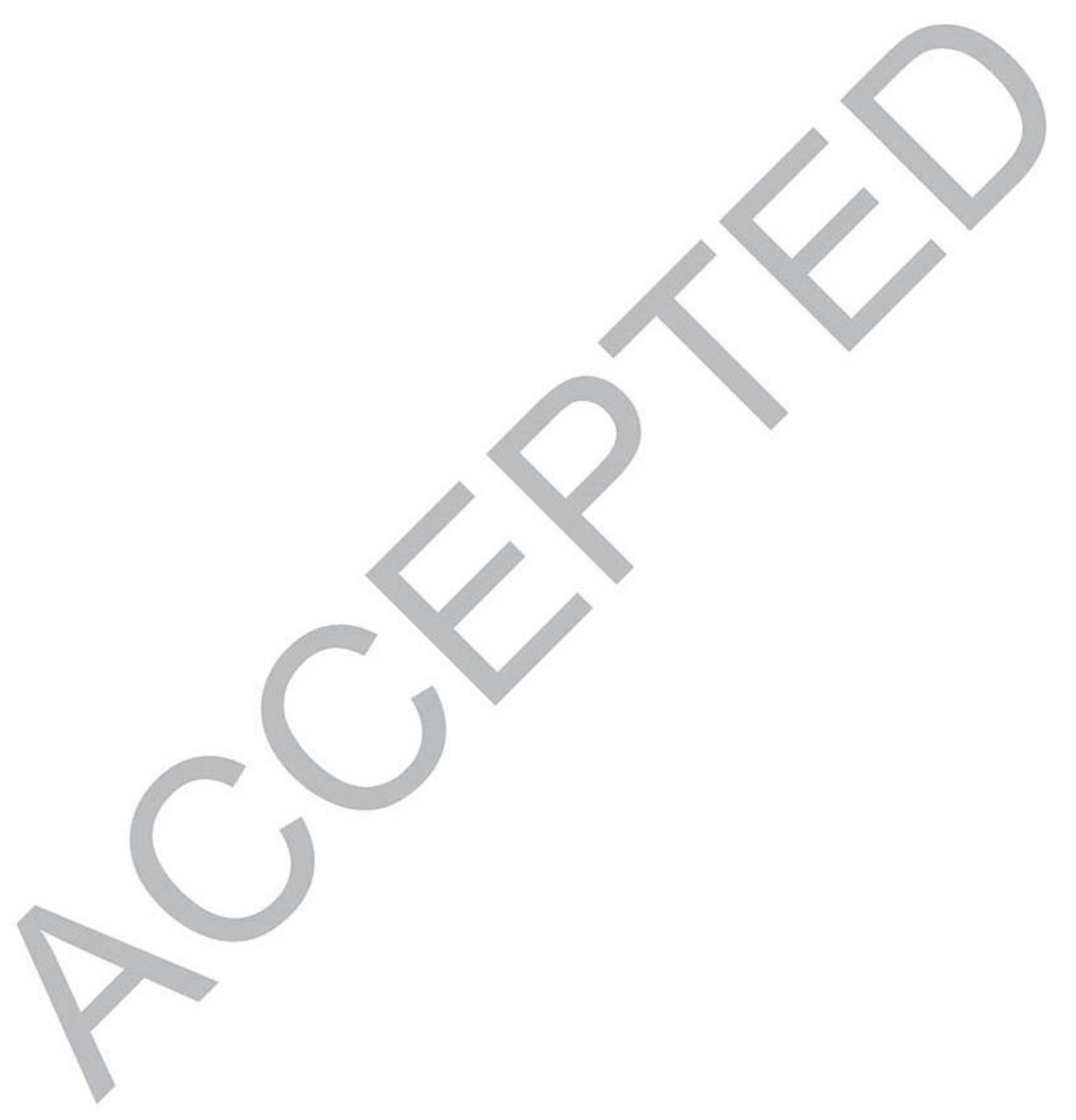

Copyright (C) 2014 Wolters Kluwer Health, Inc. Unauthorized reproduction of the article is prohibited. 


\section{References}

1. Wolfe F, Smythe HA, Yunus MB, et al. The American College of Rheumatology 1990 criteria for the classification of fibromyalgia. Report of the Multicenter Criteria Committee. Arthritis Rheumat 1990;33(2):160-172.

2. Bennett RM, Jones J, Turk DC, et al. An internet survey of 2596 people with fibromyalgia. BMC Musculoskelet Disord 2007;8:11.

3. Mease P. Fibromyalgia syndrome: review of clinical presentation, pathogenesis, outcome measures and treatment. J Rheumatol 2005; 75: 6-21.

4. Fietta P, Fietta P. Manganelli P. Fibromyalgia and psychiatric disorders. Acta Biomed 2007;78(2):88-95.

5. Spaeth M. Epidemiology, costs, and the economic burden of fibromyalgia syndrome: efficacy of operant behavioral and cognitive behavioral treatments. Arthritis Res Ther 2009;11:2.

6. Van Houdenhove B, Egle UT. Fibromyalgia: a stress disorder? Piecing the biopsychosocial puzzle together. Psychother Psychosom 2004:73:267-275.

7. García-Campayo J, Magdalena J, Magallon R, et al. A meta-analysis of the efficacy of fibromyalgia treatment according to level of care. Arthritis Res Ther 2008;10:15.

8. Hauser W, Bernardy K, Arnold B, et al. Efficacy of multicomponent treatment in fibromyalgia syndrome: a meta-analysis of randomized controlled clinical trials. Arthritis Rheum Arthritis Care Res 2009;61:216-224.

9. Turk DC, Okifuji, A. Psychological factors in chronic pain: Evolution and revolution. J Consult Clin Psychol 2002;70:678-690. 
10. Glombiewski JA, Sawyer AT, Gutermann J, et al. Psychological treatments for fibromyalgia: a meta-analysis. Pain 2010;151:280-295.

11. Powers MB, Emmelkamp P. Virtual reality exposure therapy for anxiety disorders: A metaanalysis. J Anxiety Disord 2008; 22:561-569.

12. Opris D, Pintea S, García-Palacios A, et al. Virtual reality exposure therapy in anxiety disorders: a quantitative meta-analysis. Depress Anxiety 2012;29:85-93.

13. Hoffman H, Patterson DR, Carrougher GJ, et al. Use of virtual reality for adjunctive treatment of adult burn pain during physical therapy: a controlled study. Clin J Pain 2000;16(3):244-250.

14. Malloy KM, Milling LS. The effectiveness of virtual reality distraction for pain reduction: a systematic review. Clin Psychol Rev 2010;30(8):1011-1018.

15. Patterson DR, Tininenko JR, Schmidt AE, et al. Virtual reality hypnosis: a case report. Int J Clin Exp Hypn 2004;52:27-38.

16. Patterson, DR., Hoffman, HG., García-Palacios, A, et al. Analgesic effects of posthypnotic suggestions and virtual reality distraction on thermal pain. The J Abn Psychol 2006;115(4): 834-841.

17. Keefe, FJ, Huling DA, Coggins MJ, et al. Virtual reality for persistent pain: A new direction for behavioral pain management. Pain 2012; 153(11): 2163-6.

18. Ramachandran VS, Seckel EL. Using mirror visual feedback and virtual reality to treat fibromyalgia. Med Hypotheses 2010;75:495-6.

19. Cole J, Crowle S, Austwick G, et al. Exploratory findings with virtual reality for phantom limb pain; from stump motion to agency and analgesia. Disabil Rehabil, 2009; 31(10): 846854.

Copyright (C) 2014 Wolters Kluwer Health, Inc. Unauthorized reproduction of the article is prohibited. 
20. Gaggioli A, Amoresano A, Gruppioni E, et al. A myoelectric-controlled virtual hand for the assessment and treatment of phantom limb pain in trans-radial upper extremity amputees: a research protocol. Stud Health Technol Inform [Internet] 2010;154:220-2.

21. Murray CD, Patchick E, Pettifer S, et al. Investigating the efficacy of a virtual mirror box in treating phantom limb pain in a sample of chronic sufferers. In: Paper presented at: 6th International Conference on Disability, Virtual Reality, and Associated Technology; September 18-20, 2006; Esbjerg, Denmark. Available at: <http://www.icdvrat.reading.ac.uk/2006/index2006.htm>.

22. Sato K, Fukumori S, Matsusaki T, et al. Nonimmersive virtual reality mirror visual feedback therapy and its application for the treatment of complex regional pain syndrome: an openlabel pilot study. Pain Med 2010;11(4):622-629.

23. Jeon B, Sunkun C, Lee JH. Application of virtual body swapping to patients with complex regional pain syndrome: A pilot study. Cyberpsychol, Behav Soc Netw 2014;17:366-370.

24. Gromala D, Song M, Yim J, et al. Immersive VR: a non-pharmacological analgesic for chronic pain? Proceedings of the International Conference on Human Factors in Computing Systems, 2011.

25. Oneal BJ, Patterson DR, Soltani M, et al. Virtual Reality Hypnosis In The Treatment Of Chronic Neuropathic Pain: A Case Report. Int J Clin Exp Hyp 2008; 56(4): 451-462.

26. Morris LD, Grimer-Somers KA, Spottiswoode B, et al. Virtual reality exposure therapy as treatment for pain catastrophizing in fibromyalgia patients: proof of concept study (study protocol). BMC Musculoskelet Disord 2011;12:85-92.

27. Wiederhold BK, Gao K, Sulea C, Wiederhold D. Virtual reality as a distraction technique in chronic pain patients. Cyberpsychol, Behav Soc Netw 2014;17:346-352. 
28. Botella C, Garcia-Palacios A, Vizcaíno Y, et al. Virtual reality in the treatment of fibromyalgia: a pilot study. Cyberpsych Beh Soc Net 2013; 16(3): 215-23.

29. Flor H, Turk DC. Applying the cognitive-behavioral approach to chronic pain management.

In H. Flor \& D. D. Turk (Eds.), Chronic pain. An integrated biobehavioral approach. Seattle: IASP press, 2011: 437-490.

30. Isen AM. A role for neuropsychology in understanding the facilitating influence of positive affect on social behavior and cognitive processes. In Snyder \& Lopez (Eds.), Handbook of positive psychology New York: Oxford University Press, 2002: 528-540.

31. Fredrickson BL. The role of positive emotion in positive psychology: The broaden and build theory of positive emotion. Am Psych 2001; 56: 218-226.

32. Catalino LI, Fredrickson BL. Tuesdays in the lives of flourishers: The role of positive emotional reactivity in optimal mental health. Emotion 2011; 11: 938-950.

33. Botella C, Riva G, Gaggioli A, et al. The Present and Future of Positive Technologies. Cyberpsych Behav Soc Net 2012; 15: 78-84.

34. First MB, Spitzer RL, Gibbon M, et al. Structured Clinical Interview for DSM-IV Axis I Disorders, Clinician Version (SCID-CV). Washington, D.C.: American Psychiatric Press, Inc. 1996 (Spanish version published by Masson, 1999).

35. Burckhardt CS, Clark SR, Bennet RM. The Fibromyalgia Impact Questionnaire: development and Validation. J Rheum 1991;18:728-733.

36. Esteve-Vives J, Rivera J, Salvat MI, et al. Propuesta de una versión de consenso del Fibromyalgia Impact Questionnaire (FIQ) para la población española. Reumatología Clínica 2007; 3(1):21-24

Copyright (C) 2014 Wolters Kluwer Health, Inc. Unauthorized reproduction of the article is prohibited. 
37. Cleeland CS, Ryan KM. Pain assessment: global use of the Brief Pain Inventory. Ann Ac Med Singapore 1994; 23(2): 129-138.

38. Badia X, Muriel C, Gracia A, et al. Validation of the Spanish version of the Brief Pain Inventory in patients with oncological pain. Med Clin 2003; 120(2): 52-59.

39. Jensen MP, Turner JA, Romano JM, et al. The Chronic Pain Coping Inventory: development and preliminary validation. Pain 1995;60:203-216.

40. Beck AT, Steer RA, Brown GK. Manual for the Beck Depression Inventory-II. San Antonio, TX: Psychological Corporation, 1996

41. Sanz J, Perdigón A, Vázquez C. Adaptación española del Inventario para la Depresión de Beck-II (BDI-II): 2. Propiedades psicométricas en población general. Clínica y Salud 2003; 14: $249-280$.

42. Mezzich JE, Schmolke MM. An introduction to ethics and quality of life in comprehensive psychiatric diagnosis. Psychopath 1999; 32: 119-120.

43. Borkovec TD, Nau SD. Credibility of analogue therapy rationales. J Behav Ther Exp Psychiatry, 1972;3:257-260.

44. Baños RM, Liaño V, Botella C, et al. Changing Induced Moods via Virtual Reality. In W. Ijsselsteijn, Y. de Kort, C. Midden, B. Eggen, E. van den Hoven, (Eds.), Persuasive Technology: Lecture Notes in Computer Science Berlin/Heilderberg: Springer-Verlag, 2006: 7-15.

45. Baños RM, Guillen V, Quero S, et al. A virtual reality system for the treatment of stressrelated disorders: A preliminary analysis of efficacy compared to a standard cognitive behavioural program. Int J Hum Comp Stud 2011; 69: 602-613 
46. Botella C, García-Palacios A, Guillen V, et al. An adaptive display for the treatment of diverse trauma PTSD victims. Cyberpsychol Beh Soc Net 2010; 13(1): 67-71.

47. Herrero R, Garcia-Palacios A, Castilla D, Molinari G Botella C. Virtual reality for the induction of positive emotions in the treatment of fibromyalgia: A pilot study over acceptability, satisfaction, and the effect of virtual reality on mood. Cyberpsychol Beh Soc Net 2014; 17:379-384.

48. Balkwill L, Thompson WF. A cross-cultural investigation of the perception of emotion in music: Psychophysical and cultural cues. Music Percep 1999; 17:43-64.

49. Gabrielsson A, Lindstrom E. The influence of musical structure on emotional expression. In: Juslin, P.N., Sloboda, J.A. (Eds.), Music and Emotion: Theory and Research Oxford: Oxford University Press, 2001:223-248.

50. Hevner K. The affective character of the major and minor mode in music. Am J Psychol 1935; 47: 103-118.

51. Rigg MG. Speed as a determiner of musical mood. J Exp Psychol 1940; 27: 566-571.

52. Bradley MM, Lang PJ. International affective digitized sounds (IADS): Stimuli, instruction manual and affective ratings. Technical report B-2. Gainesville, FL: The Center for Research in Psychophysiology, University of Florida, 1999.

53. Smith CA, Ellsworth PC. Patterns of cognitive appraisal in emotion. J Pers Soc Psychol $1985 ; 48: 813-838$

54. Yik MSM, Russell JA, Barrett LF. Structure of self-reported current affect: Integration and beyond. J Pers Soc Psychol 1999; 77: 600-619. 
55. Lang PJ, Bradley MM, Cuthbert BN. International affective picture system (IAPS): Technical manual and affective ratings. Gainesville, FL: The Center for Research in Psychophysiology, University of Florida, 1995.

56. Moltó J, Montañés S, Poy R, et al. Un nuevo método para el estudio experimental de las emociones: El International Affective Pictures System (IAPS). Adaptación española. Rev Psicol Gen Aplic 1999; 52: 55-87.

57. Guilford JP, Smith PC. A system of color-preferences. Am J Psychol 1959; 72: 487-502.

Copyright (C) 2014 Wolters Kluwer Health, Inc. Unauthorized reproduction of the article is prohibited. 
Table 1. Mean and standard deviation for each variable at pretreatment and posttreatment and Cohen $\mathrm{d}$ for each comparison.

\begin{tabular}{|c|c|c|c|c|}
\hline Variable & Condition & $\begin{array}{c}\text { Pretreatment } \\
M \text { and } S D\end{array}$ & $\begin{array}{c}\text { Posttreatment } \\
M \text { and } S D\end{array}$ & Cohen $d$ \\
\hline \multirow[t]{2}{*}{ FIQ } & VRAM & $61.59(19.91)$ & $42.37(15.68)$ & 1.07 \\
\hline & TAU & $60.57(21.38)$ & $57.02(17.49)$ & 18 \\
\hline \multirow[t]{2}{*}{ BDI-II } & VRAM & $19.90(10.68)$ & $16.55(8.52)$ & 0.35 \\
\hline & TAU & $20.53(10.16)$ & $19.67(9.95)$ & 0.08 \\
\hline \multirow[t]{2}{*}{ BPI: Intensity } & VRAM & 23.55 (5.07) & $2.61(6.25)$ & 0.17 \\
\hline & TAU & 22.37 (7.97) & $0.70(8.32)$ & 0.21 \\
\hline \multirow[t]{2}{*}{ BPI: Interference } & VRAM & $32.19(14.80)$ & $31.23(17.66)$ & 0.06 \\
\hline & TAU & $32.33(16.68)$ & $37.07(12.93)$ & -0.56 \\
\hline \multirow[t]{2}{*}{ QLI } & VRAM & $4.50(1.24)$ & $6.10(1.42)$ & -1.20 \\
\hline & TAU & $5.04(1.22)$ & $5.26(1.33)$ & -0.17 \\
\hline \multicolumn{5}{|l|}{ CPCI } \\
\hline \multirow[t]{2}{*}{ Guarding } & & $3.43(1.36)$ & $2.63(0.97)$ & 0.68 \\
\hline & & $3.57(0.96)$ & $3.42(1.35)$ & 0.13 \\
\hline \multirow[t]{2}{*}{ Resting } & VRAM & $3.77(1.52)$ & $3.32(1.35)$ & 0.31 \\
\hline & TAU & $4.00(1.55)$ & $4.34(1.30)$ & -0.23 \\
\hline Asking for & VRAM & $2.34(1.33)$ & $2.69(1.51)$ & -0.25 \\
\hline assistance & TAU & $2.79(1.75)$ & $2.93(1.65)$ & -0.08 \\
\hline Relaxation & VRAM & $2.22(1.18)$ & $2.84(1.45)$ & -0.47 \\
\hline
\end{tabular}

Copyright (C) 2014 Wolters Kluwer Health, Inc. Unauthorized reproduction of the article is prohibited. 


\begin{tabular}{lllll} 
& TAU & $2.28(1.67)$ & $2.53(1.67)$ & -0.15 \\
Task persistence & VRAM & $3.74(1.70)$ & $5.07(1.32)$ & -0.87 \\
& TAU & $3.97(1.19)$ & $4.03(1.34)$ & -0.05 \\
\multirow{2}{*}{ Exercise } & VRAM & $2.87(1.46)$ & $3.94(1.78)$ & -0.65 \\
& TAU & $3.17(1.68)$ & $3.15(1.77)$ & 0.01 \\
\multirow{2}{*}{ Coping self- } & VRAM & $3.01(1.51)$ & $3.92(1.79)$ & -0.55 \\
statements & TAU & $2.97(1.74)$ & $3.32(1.53)$ & -0.48 \\
Seeking social & VRAM & $2.48(1.42)$ & $2.69(1.46)$ & -0.49 \\
support & TAU & $2.91(2.00)$ & $2.54(1.45)$ & 0.29 \\
\hline
\end{tabular}

Cohen (1988) defines as $d=0.2$ are regarded as a "small" effect size, $d=0.5$ as "medium," and $d=0.8$ as "large."

FIQ: Fibromyalgia Impact Questionnaire; BDI-II: Beck Depression Inventory; BPI: Brief Pain Inventory; QLI: the Quality of Life Index; CPCI: Chronic Pain Coping Inventory; VRAM: Virtual reality activity management; TAU:

Treatment as usual.

Copyright (C) 2014 Wolters Kluwer Health, Inc. Unauthorized reproduction of the article is prohibited. 
Table 2. Mean and standard deviation of the ratings given by the patients regarding their satisfaction with the treatment program and with the use of VR at posttreatment.

\section{Satisfaction and Acceptability Questionnaire}

M (DT)

How logical did the treatment seem to you?

$8.45(1.52)$

How satisfied are you with the treatment?

$8.83(1.39)$

To what extent would you recommend this treatment to a friend?

$9.07(1,13)$

How useful was this treatment for you?

$9.00(1,10)$

How unpleasant was the treatment for you?

$0.86(2,35)$

How satisfied are you with the VR component?

$9.00(1,08)$

How useful was the VR component to you?

$9.08(0.98)$

Copyright (C) 2014 Wolters Kluwer Health, Inc. Unauthorized reproduction of the article is prohibited. 
Figure 1. Study Flow diagram

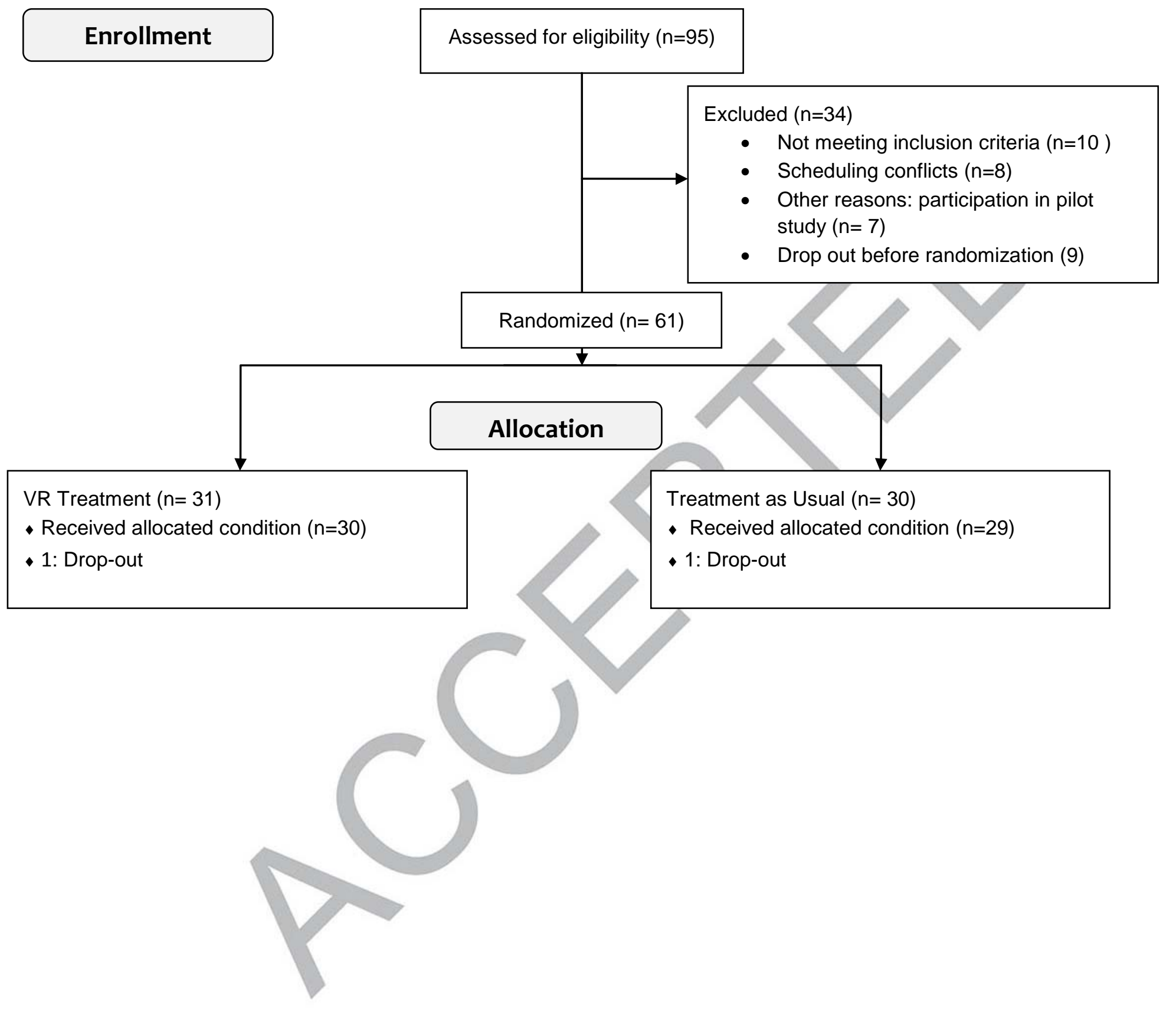

Copyright (C) 2014 Wolters Kluwer Health, Inc. Unauthorized reproduction of the article is prohibited. 
Click here to download high resolution image

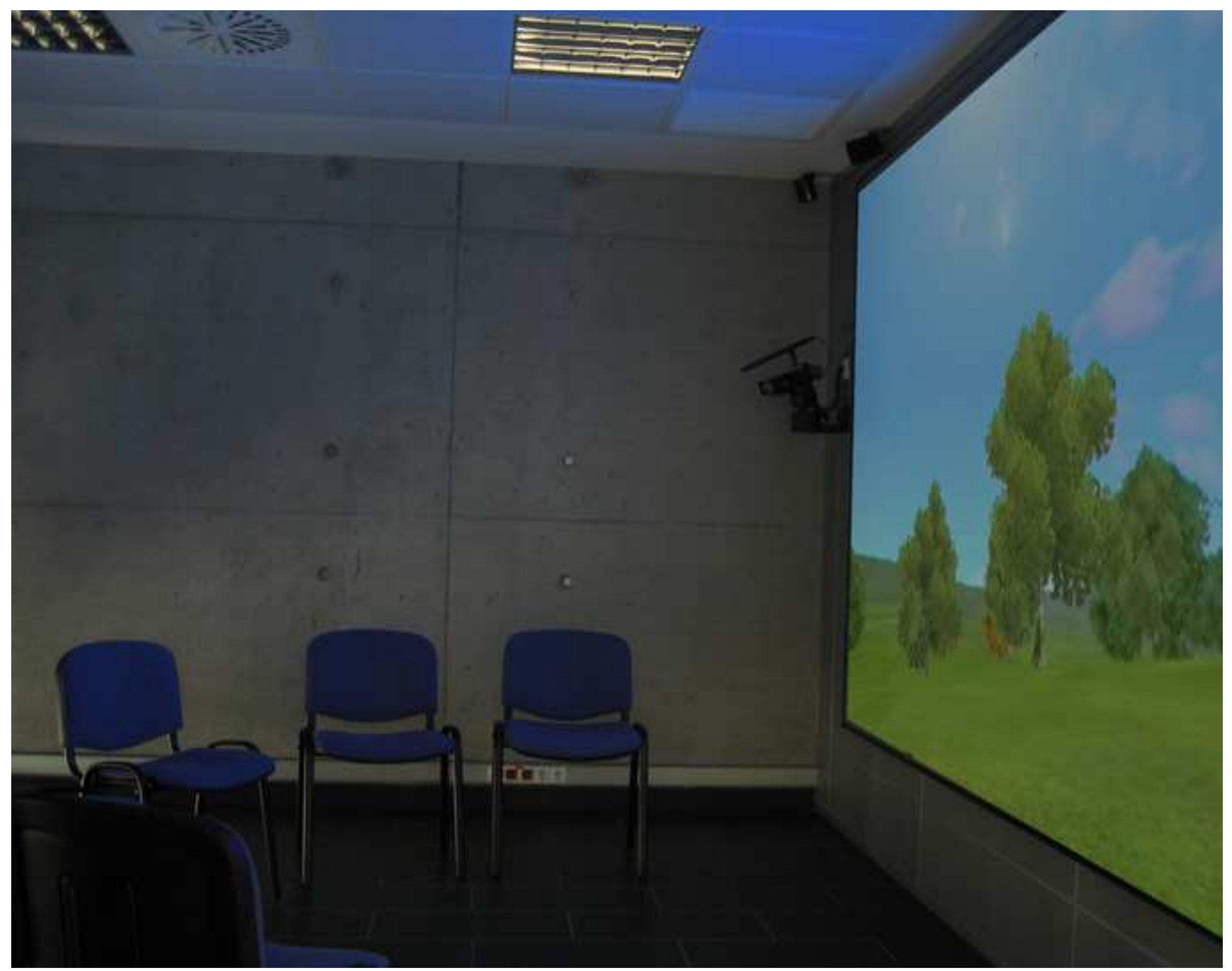

Copyright (C) 2014 Wolters Kluwer Health, Inc. Unauthorized reproduction of the article is prohibited. 


\section{THE IMPOSSIBLE JUST TAKES A LITTLE MORE}

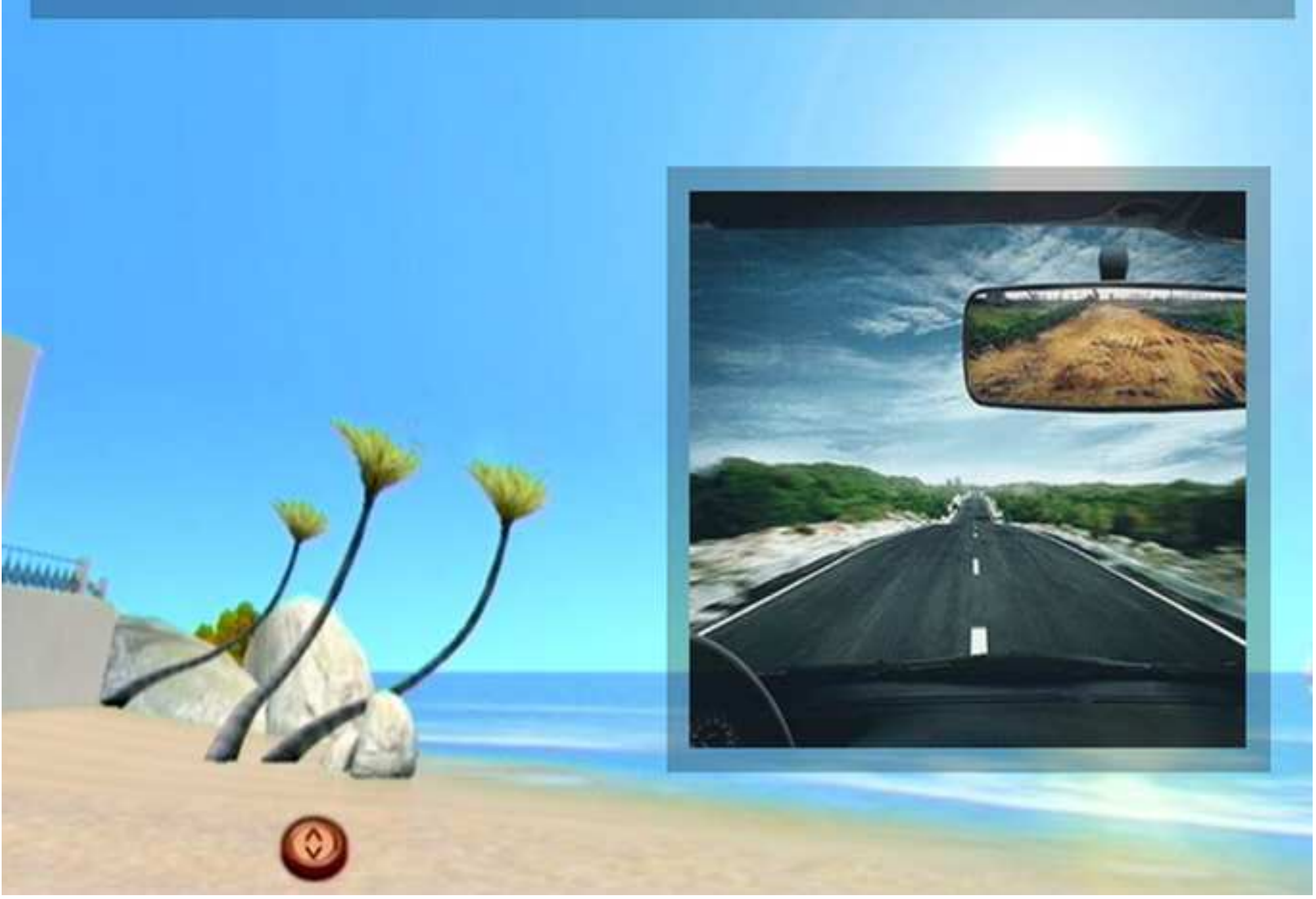

Copyright (C) 2014 Wolters Kluwer Health, Inc. Unauthorized reproduction of the article is prohibited. 